\title{
Regarding "Rate and Prognosis of Patients under Conscious Sedation Requiring Emergent Intubation during Neuroendovascular Procedures"
}

W e read with interest "Rate and Prognosis of Patients under Conscious Sedation Requiring Emergent Intubation during Neuroendovascular Procedures" ${ }^{1}$ and appreciate the effort of Hassan et al to address the complicated, controversial issue of conscious sedation versus general anesthesia for neurointerventional procedures. As they appropriately noted, "Limitations of the methodology used in our study preclude us from offering specific recommendations regarding when to use a specific anesthetic protocol."

Although Hassan et al $^{1}$ clearly declare the limitations of their study, they nevertheless present a false equivalence. They assert as favorable their finding that the clinical outcomes were similar between patients who were selected for treatment under general anesthesia and patients who were selected for conscious sedation but emergently converted to general anesthesia. While the outcomes for these 2 groups may be similar, the treatment groups clearly are not. The patients selected for general anesthesia were patients with more complex pathology and higher NIHSS scores. The largest groups of patients selected for conscious sedation were patients with extracranial pathology — carotid stenosis-or the unorthodox group of "AVM/epistaxis." Given this preprocedural selection bias, one would have expected patients with the more straightforward pathology, better NIHSS score, and conscious sedation conversion to have better outcomes than the patients with more complex pathology and higher NIHSS scores. We do not know why the patients in this study needed emergent conversion to general anesthesia and are left wondering whether adequate anesthesia and control of patient motion might have prevented some of the events that led to this conversion and the resulting worsened outcomes. Similarly, regarding the group that remained under conscious sedation, there is no way to know whether that group was well-served with the choices made or whether better outcomes would have been seen with a higher or lower proportion of conscious sedation versus general anesthesia.

Experienced interventionalists recognize that during endovascular procedures, uncontrolled patient motion at key moments can be catastrophic. Ready examples include uncontrolled movement following (or precipitating) procedural rupture of intracranial aneurysms and undesired movement that impairs visibility during injection of liquid adhesives.

What we can glean from this study is that at a single institution, without well-defined criteria for selecting conscious sedation versus general anesthesia, the decision to convert to general anesthesia was probably associated with poorer outcomes than expected, but it is entirely unclear whether the outcomes could have been improved by altering (in either direction) the proportion of patients selected for conscious sedation versus general anesthesia.

The question is not whether negative consequences occur from patient motion-they do. The question is whether the negative consequences of general anesthesia outweigh the benefits. Undoubtedly, the appropriate anesthetic choice is case-dependent, so the issue becomes defining clear objective criteria that can help us select cases appropriately. Unfortunately, we are no closer to resolving this issue.

In drawing conclusions from a study, no amount of careful statistical analysis can overcome the effects of overt selection bias. While retrospective studies comparing dissimilar groups may help to gather useful information for designing a trial to address this important issue in a logical way, it is not helpful to present, unchallenged, a fundamentally flawed analysis. While Hassan et $\mathrm{al}^{1}$ are to be commended for their interest and effort in this matter, the editors have a responsibility to present to their readers studies that do not obfuscate clinical dilemmas with interpretations unsupported by the data presented.

\section{REFERENCE}

1. Hassan AE, Akbar U, Chaudhry SA, et al. Rate and prognosis of patients under conscious sedation requiring emergent intubation during neuroendovascular procedures. AJNR Am J Neurradiol 2013 Jan 31. [Epub ahead of print]

C.G. McDougall F.C. Albuquerque

A.F. Ducruet

Division of Neurological Surgery Barrow Neurological Institute St. Joseph's Hospital and Medical Center 